\title{
PALLIATIVE CARE IN BREAST CANCER: CHALLENGES IN MEDICAL PRACTICE AND PROMOTING QUALITY OF LIFE
}

Silvaleide Ataides Assunção', lanca Leandra Santos², Rosemar Macedo Sousa Rahal ${ }^{3}$

${ }^{1}$ Faculdade de Medicina, Universidade Federal de Goiás - Goiânia (GO), Brazil. 2Faculdade Unida de Campinas - Goiânia (GO), Brazil.

3Hospital das Clínicas, Universidade Federal de Goiás - Goiânia (GO), Brazil.

Objectives: Breast cancer is the main neoplasm affecting women, and in many cases, curative treatment is not feasible. In this context, it is important to work with other forms of assistance that provide symptom relief and better quality of care for these patients. Therefore, the objective of this work is to portray the importance of attention and care to patients with breast cancer, whose cure is not possible, since it generates better acceptance of the situation and a more humanized end, with less suffering, pain, and anguish. Methodology: This is a descriptive research carried out based on a review of the medical literature available through a survey of publications from the past 12 years in the PubMed, Lilacs, and MedLine databases. The following descriptors were used: palliative care, home care, and breast cancer. Results: From the analysis performed, it can be seen that approximately $30 \%$ of patients diagnosed with breast cancer have some depressive disorder. This fact occurs due to the shock of the news of having an extremely serious disease, due to the disorders arising from the treatment or the progression of the disease itself. When assessing patients with breast cancer, who have palliative care at home, this percentage drops to approximately 9\%, meaning an abrupt improvement in the quality of life of these people, resulting from multiprofessional home treatment. Conclusion: Given this situation, it is observed that home care, though insufficiently explored, has a good response for terminally ill patients. Therefore, it appears that palliative care should be better explored by both the public and private health systems, as this tool is capable of mitigating the adversities caused by cancer and improving the quality of life of patients and their families in this difficult stage.

Keywords: Palliative Care; Home Care; Breast Cancer. 